\title{
On Neural Network Modeling of Main Steam Temperature for Ultra - supercritical Power Unit with Load Varying
}

\author{
Xifeng Guo a, Jingtao Huang, Lijie Wang, Jia Zhang \\ Electrical Engineering College, Henan University of Science and Technology, Luoyang 471023, \\ P.R.China \\ aguoxfly@163.com
}

\begin{abstract}
Keywords: Ultra-supercritical unit; Main steam temperature; Operation data; Neural network; Modeling

Abstract. In order to solve the problem of accurate modeling of main steam temperature in ultra-supercritical (USC) unit under variable load conditions, the BP neural network model was established based on the field operation data. Considering the operation mechanism of the process, combing the relevant analysis of the sampled parameters, the feature parameters were chosen as the model inputs, the selected data was scaled to avoid the influence of different parameters range, then a 3 layer BP neural network model was established. By changing the number of hidden layer nodes in the neural network and the type of the hidden and the output layer activation function, the influence on the gained model performances under the variable load conditions was investigated. The simulation results on a $1000 \mathrm{MW}$ unit show that the model can reveal the main steam temperature changes in the actual production process.
\end{abstract}

\section{Introduction}

With the increasingly severe situation of energy saving and emission reduction, in order to improve the thermal efficiency of thermal power plant, the designed steam parameters are gradually increased, the security issues become more prominent ${ }^{[1]}$. Boiler is one of the most important equipment in thermal power plant. Main steam temperature is one of the main indicators of boiler operation quality. The adjustment of main steam pressure, temperature, etc. has more obvious impact on the economy and security. In the actual plant operation, it is necessary to achieve stable control of the main steam temperature and to maintain the outlet temperature of the superheater in the required range, for improving the economic efficiency and ensuring the safe operation of the unit. If the steam temperature is too high, it will destroy the superheater and steam turbine. If the steam temperature is too low, the thermal efficiency will decrease, and the steam humidity of last few steam turbine blades may increase which results in the increasing turbine blade erosion. With the rapid change of steam temperature, the metal parts will have too much thermal stress and thermal deformation, or even the friction between static components and dynamic components. Therefore, we must strictly control the main steam temperature so that the temperature is in the vicinity of the given value. The main influencing factors that can affect the main steam temperature include boiler load, feed water temperature, desuperheating water temperature, fuel quantity and total air volume ${ }^{[2]}$. The main steam temperature control is a hard task with the characteristics of large inertia and large delay. In [3], the USC boiler unit is analyzed through the mechanism modeling, by comparing with the intermediate point temperature, the main steam temperature characteristic, the heat absorption ratio and the coal quality change, water-coal ratio control. The experimental results show that the change of coal quality is the main adjustment factor of water-coal ratio, and the change of radiation and convective heat transfer ratio has great influence on boiler operation characteristics. In [4], the main control loop of the conventional PID control system is added to the dynamic matrix predictive controller to form the cascade control of the main steam temperature, and the performance of the control system is improved. Many researchers use different algorithms to model the process with field data, which avoids the complexity of mathematical modeling and the implement difficulty, it has a strong applicability. In [5], an improved harmonic search algorithm combined with particle swarm optimization algorithm was proposed to identify the main steam temperature system model. In [6], the support vector regression 
model was used to model based on the field data. In [7], the neural network model was established to predict the wall temperature of the supercritical boiler, and the wall materials with appropriate heat resistance were selected to ensure the safety of the boiler operation. In [8], the superheater temperature model of a 1000MW supercritical unit was studied by BP, RBF and Elman neural network respectively. In [9], based on data-driven and fuzzy modeling strategy, the operating range of the boiler was divided into sub-spaces, and the model was analyzed in each subspace, which makes it more accurate. However, when the load changes large and frequently, it will increase the control difficulty with the nonlinear and time-varying characteristics of the superheated steam temperature. The mechanism modeling which relys on technological process often makes some approximations and assumptions when it comes to mathematical derivation. Although these assumptions have some theoretical basis, they cannot fully reflect the actual conditions. But the data derived from the actual production process often imply the internal relationship of the production process. How to use the industrial process data to improve the operation performance has become a big issue. A data-based neural network modeling method is proposed to model the main steam temperature with frequent load varying.

\section{Dynamic Characteristics of Main Steam Temperature on USC Unit}

In USC unit, steam from the separator goes through superheater in the furnace and exchanges heat with high temperature flue gas. The steam temperature of the final stage superheater outlet is main steam temperatures that need to be well controlled. Fig. 1 shows the boiler steam-water flow.

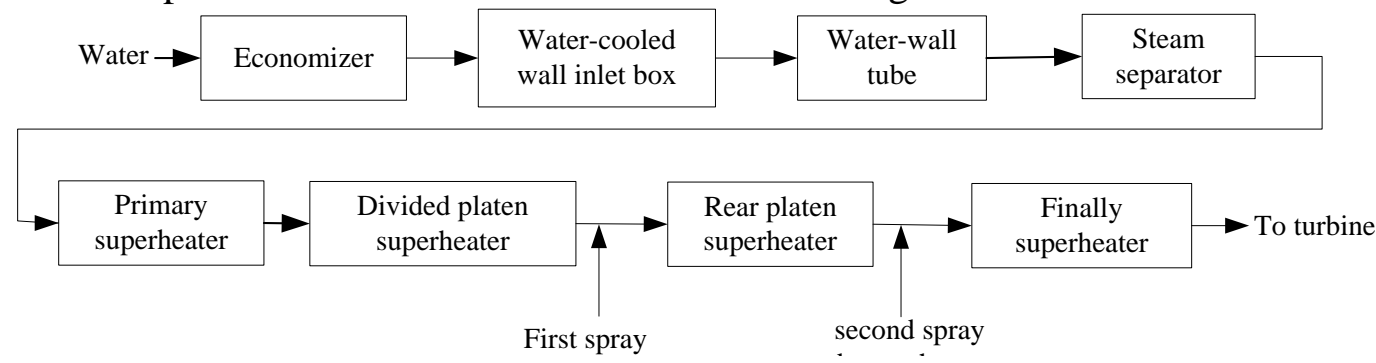

Fig.1. Steam-water flow in USC unit

Since the main steam temperature has the characteristics of large inertia, large delay and strong coupling, and the traditional simplified mechanism model cannot satisfy the control demand in the wide range of variable working conditions. While the data acquisition system of the unit has accumulated a large amount of data, establishing the main steam temperature model based on the operational data is an effective method to solve the accurate modeling of the main steam temperature.

\section{Main Steam Temperature Modeling Based on Neural Network}

The usual mechanism modeling simplifies the model parameters greatly, so the modeling accuracy can be hardly guaranted. Neural network modeling just need to consider the input and output of the system, so it is convenient to be modeled. McCulloch and Pitts proposed a single neuron mathematical description in $1943^{[10]}$. Neural network has a strong nonlinear fitting ability, self-learning ability and self-organization ability.

The principle of Neural Network Algorithm. Aritifical neural network(ANN) consists of many interconnected artificial neurons with linear or nonlinear transfer functions, and can predict the nonlinear behavior of the system. The error back propagation (BP)neural network in the multilayer feedforward network is the type of network used in this study.

Fig. 2 shows a typical three-layer BP network structure. As shown in equation (1), typically, each output of the neural network can be represented by this expression. In general, a neural network consists of one input layer, one output layer and one or more hidden layers. Several signals $x_{j}(j=$ $1, \ldots, \mathrm{m})$ are supplied to the neural as input. Each input signal is multiplied by their weight $\mathrm{W}_{\mathrm{i}, \mathrm{j}}$ and then summed up with threshold $\theta_{\mathrm{i}}(\mathrm{i}=1, \ldots, \mathrm{q})$. The sum represents the argument of the neuron activation function $\Phi$. It can be further transferred to another layer neurons, where it represents one 
of input signals. The result of the output layer activation function is the output of the whole network.

$$
o_{k}=\psi\left(\sum_{k=1}^{L} w_{k, i} \phi\left(\sum_{i=1}^{q} w_{i, j} x_{j}\right)\right) .
$$

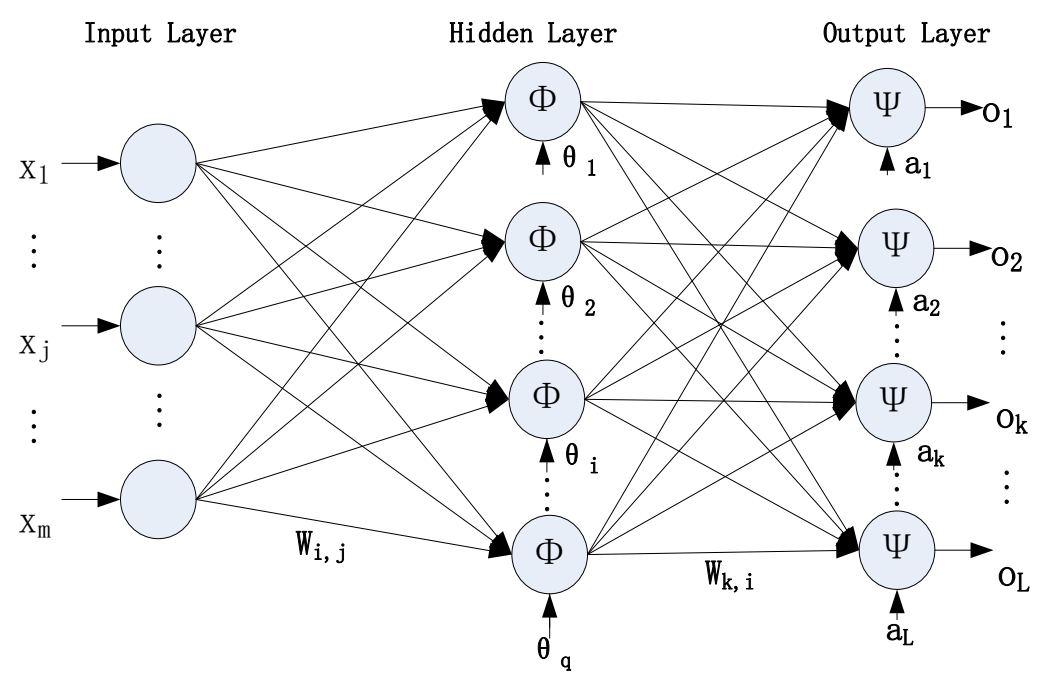

Fig.2. Diagram of typical three layer BP network

Main Steam Temperature Modeling on BP Network. There is a complex nonlinear relationship between the main steam temperature and the feature parameters. The data accumulated in the operation process contains inherent laws. We can get a good training result and save the entire training time with an appropriate data set even for a complex system. Therefore, the selection of parameters is important. The direct and indirect factors influencing the main steam temperature were selected by analyzing the operation mechanism of the $1000 \mathrm{MW}$ coal-fired generating. The relative parameters, including coal supply, secondary air ratio, water supply, OFA etc. were chosen as the model inputs. The feature parameters for the main steam temperature modeling are shown in Table 1.

Table 1 Feature parameters for the main steam temperature modeling

\begin{tabular}{|l|c|c|c|c|c|}
\hline Feature parameters & unit & $14: 20$ & $14: 25$ & $14: 30$ & $14: 35$ \\
\hline Economizer inlet water flow & $\mathrm{t} / \mathrm{h}$ & 2990.21 & 2992.07 & 2992.74 & 2975.18 \\
\hline Economizer inlet water temperature & ${ }^{\circ} \mathrm{C}$ & 298.14 & 298.14 & 297.93 & 298.14 \\
\hline Boiler feed water pressure & $\mathrm{Mpa}$ & 30.3 & 30.3 & 30.3 & 30.3 \\
\hline A-side economizer outlet temperature & ${ }^{\circ} \mathrm{C}$ & 338.06 & 338.06 & 338.06 & 338.06 \\
\hline B-side economizer outlet temperature & ${ }^{\circ} \mathrm{C}$ & 336.53 & 336.53 & 336.53 & 336.53 \\
\hline superheater outlet pressure & $\mathrm{Mpa}$ & 26.28 & 26.28 & 26.28 & 26.28 \\
\hline B-side primary superheater flow & $\mathrm{t} / \mathrm{h}$ & 51.54 & 48.86 & 40.84 & 36.91 \\
\hline A-side second superheater flow & $\mathrm{t} / \mathrm{h}$ & 156.54 & 156.54 & 156.54 & 156.54 \\
\hline A supply fan inlet temperature & ${ }^{\circ} \mathrm{C}$ & 21.56 & 21.56 & 21.56 & 21.61 \\
\hline Total air volume & $\mathrm{t} / \mathrm{h}$ & 2815.43 & 2886.56 & 2845.02 & 2887.7 \\
\hline OFA of A front wall & $\mathrm{t} / \mathrm{h}$ & 233.14 & 234.44 & 234.88 & 240.58 \\
\hline OFA of A rear wall & $\mathrm{t} / \mathrm{h}$ & 194.88 & 197.86 & 190.16 & 209.85 \\
\hline Feedback of coal feeder A & $\mathrm{t} / \mathrm{h}$ & 76.96 & 77.56 & 77.54 & 77.54 \\
\hline Feedback of coal feeder B & $\mathrm{t} / \mathrm{h}$ & 77.26 & 77.26 & 77.26 & 77.26 \\
\hline Feedback of coal feeder C & $\mathrm{t} / \mathrm{h}$ & 66.07 & 66.1 & 66.67 & 66.67 \\
\hline Feedback of coal feeder D & $\mathrm{t} / \mathrm{h}$ & 76.17 & 76.17 & 76.79 & 76.79 \\
\hline Feedback of coal feeder E & $\mathrm{t} / \mathrm{h}$ & 77.61 & 77.61 & 78.19 & 78.2 \\
\hline Feedback of coal feeder F & $\mathrm{t} / \mathrm{h}$ & -0.18 & -0.18 & -0.18 & -0.18 \\
\hline
\end{tabular}




\section{Simulation and Analysis}

In this paper, the model was established in MATLAB R2013a neural network toolbox environment. Many factors can affect the modeling results, including input and output parameters, the structure of neural network, the type of the transfer function, the number of hidden layers and neuron nodes etc. Feature Selection and Data Preprocessing. Considering the operation mechanism of the process, combing the relevant analysis of the sampled parameters, the feature parameters were chosen as the Table 1. A data set consisting of 338 samples charactering the operation of the boiler collected in 5 minutes interval were used to carry out the simulation. The whole set was seperated randomly into training and test set, $270(80 \%)$ points of the whole set were used to train the model and the remaining were used to test. The load and main steam temperature variation are shown in Fig. 3 (a) and (b).

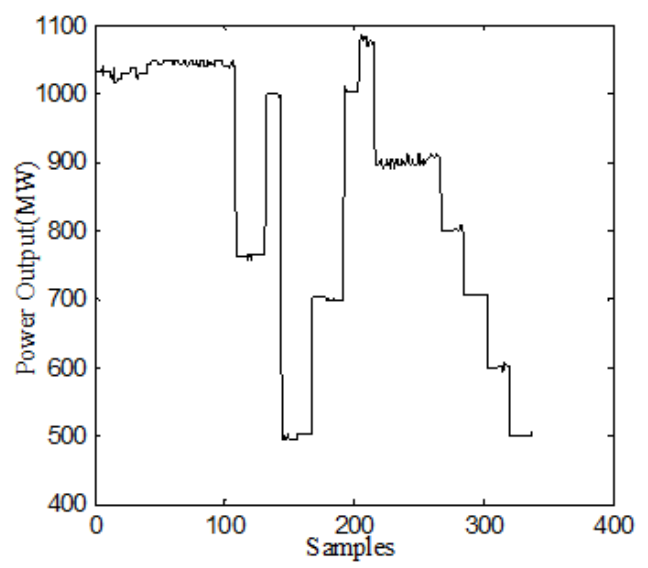

(a) Load variation

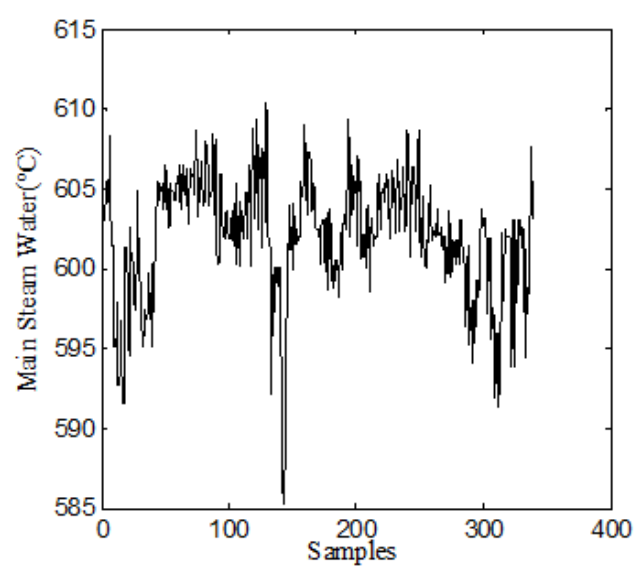

(b) The main steam temperature variation

Fig.3.The varying load operation condition

the boiler characteristics vary with the load, to improve model performance, the load of the training sample should cover the whole operating range of the experimental conditions. The sample data were normalized to $[0,1]$ before training to avoid the numerical influence among different parameters. The normalization is implemented according to equation (2). Where $\mathrm{x}$ and $\mathrm{x}$ ' are the values before and after the normalization, respectively.

$$
x^{\prime}=\frac{x-x_{\min }}{x_{\max }-x_{\min }} .
$$

Main Steam Temperature Modeling Simulation. Theoretically, neural network can approximate any nonlinear mapping relationship as long as there are enough hidden layers and nodes. However, the number of hidden layers and hidden nodes is difficult to determine which usually relies on experience. To make the prediction output better fit the expected value, we choose the single hidden layer neural network to test the hidden node number and explore its influence on the main steam temperature output value. According to experience, the optimal number of hidden layer nodes is chosen as: $l<n-1, l<\sqrt{(m+n)}+a, l=\log _{2} n$, Where $n, l, m$ is the number of nodes in the input layer, hidden layer and output layer, respectively. And $a$ is a constant between 0 and 10 . Therefore, the number of hidden layers is between 4 and 20. Table 2 shows the relative error and MSE varying with the different number of hidden layer nodes. Fig.4 shows the relative error of the output main steam temperature predicted by neural network. The figure shows that the number of hidden layer nodes has influence on the accuracy of the prediction performance with irregularity. It has the best prediction result with the smallest MSE when the number of hidden neurons $l$ equal 12. Fig.5 shows the different MSE of the predicted values and target value of the main steam temperature with the number of hidden nodes changes. Fig. 6 shows the absolute error comparison of neural network prediction results when the number of hidden nodes is 8 and 12 respectively. It can be seen that when the number is 12 , both the maximum error and error range are better than 8 hidden nodes. 
Table 2 Test error with different hidden layer nodes

\begin{tabular}{|l|c|c|c|c|c|c|c|c|c|c|c|c|c|c|c|c|c|c|}
\hline HiddenNodes & 4 & 5 & 6 & 7 & 8 & 9 & 10 & 11 & 12 & 13 & 14 & 15 & 16 & 17 & 18 & 19 & 20 & 21 \\
\hline Relative Error & 0.38 & 0.36 & 0.36 & 0.35 & 0.33 & 0.37 & 0.36 & 0.37 & 0.31 & 0.36 & 0.35 & 0.36 & 0.38 & 0.36 & 0.38 & 0.34 & 0.35 & 0.37 \\
\hline MSE & 7.4 & 7.61 & 7.35 & 7.02 & 7.59 & 8.32 & 7.16 & 7.45 & 6.33 & 7.41 & 7.93 & 7.64 & 7.85 & 7.82 & 7.47 & 6.84 & 7.47 & 7.75 \\
\hline
\end{tabular}

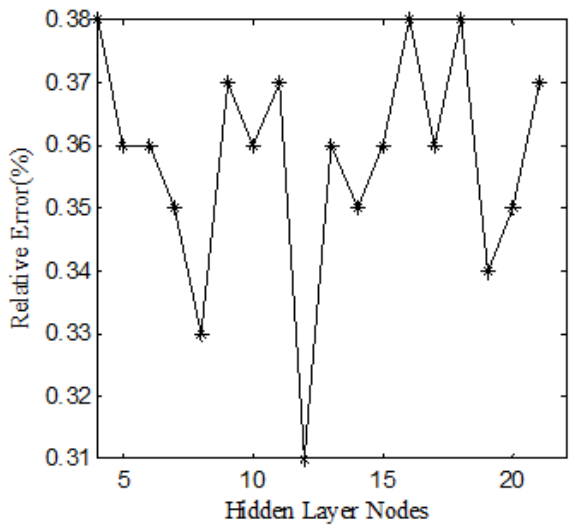

Fig.4.Prediction error with different hidden nodes

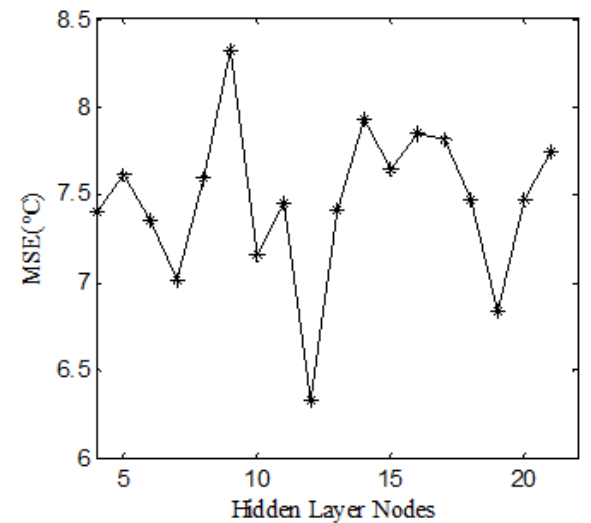

Fig.5. MSE with different hidden nodes

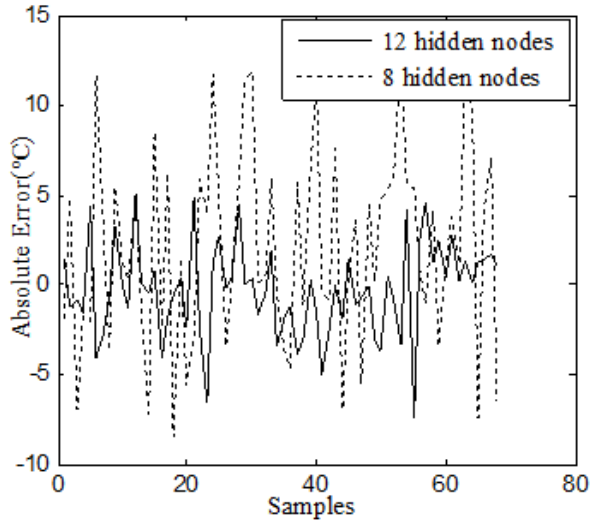

Fig.6. Absolute error of prediction

Sigmoid function is often used as BP neural network activation function, including tansig, logsig, and purelin function, as the equation (3) shows. This article carried out the experiment with three forms of activation function, the result is shown in Table 3.

Table. 3 Prediction error with different activation function

\begin{tabular}{|c|c|c|c|}
\hline $\begin{array}{c}\text { Hidden Layer } \\
\text { Function }\end{array}$ & $\begin{array}{c}\text { Hidden Layer } \\
\text { Function }\end{array}$ & $\begin{array}{c}\text { Relative } \\
\text { Error }\end{array}$ & MSE \\
\hline logsig & logsig & 0.41 & 8.75 \\
\hline logsig & tansig & 0.38 & 8.7 \\
\hline logsig & purelin & 0.39 & 8.86 \\
\hline tansig & logsig & 0.31 & 6.33 \\
\hline tansig & tansig & 0.37 & 8.78 \\
\hline tansig & purelin & 0.39 & 8.31 \\
\hline pulelin & logsig & 0.36 & 8.74 \\
\hline purelin & tansig & 0.36 & 8.63 \\
\hline purellin & purelin & 0.39 & 9.72 \\
\hline
\end{tabular}

$$
y=\left\{\begin{array}{lr}
\frac{1}{1+e^{-x}}, & \text { tansig } \\
\frac{2}{1+e^{-2 x}}-1 & \text { logsig } \\
x, & \text { purelin }
\end{array}\right.
$$




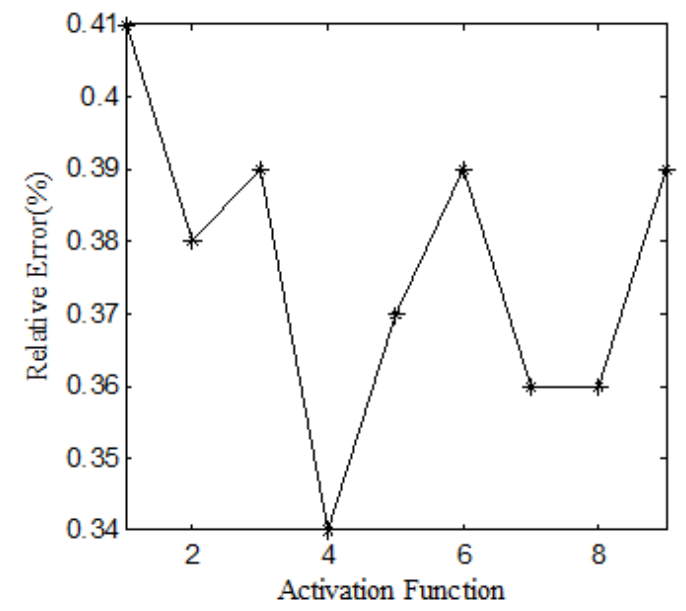

(a) Prediction error

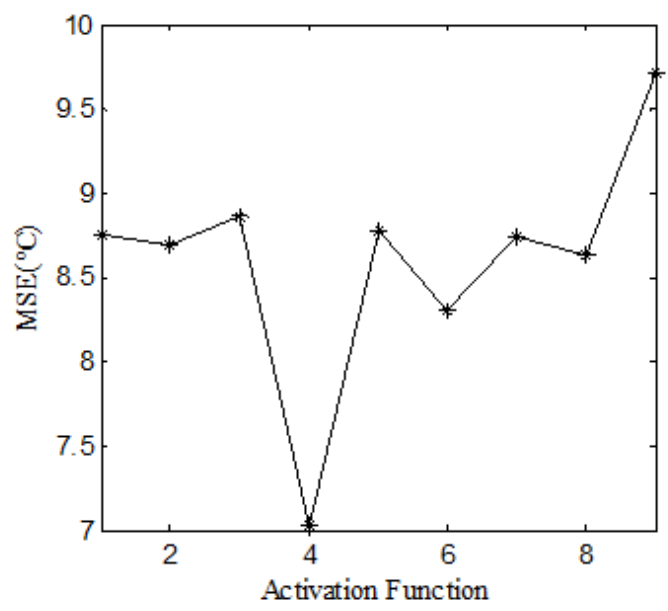

(b) MSE

Fig.7. Prediction performance with different activation function

Fig.7 (a) shows the relative error between the prediction and the target value. Fig.7 (b) shows the influence of the different activation function types on MSE. From Table 3 and Figure 7, it can be seen that different combinations of activation function makes influence of the prediction performance different. When the hidden layer activation function takes tansig, and the output layer activation function takes logsig or tansig, the prediction is better. In summary, we selected 12 hidden layer nodes, the hidden layer and output layer activation function were tansig and logsig, modeling on the main steam temperature, the testing and training results are shown in Fig.8.

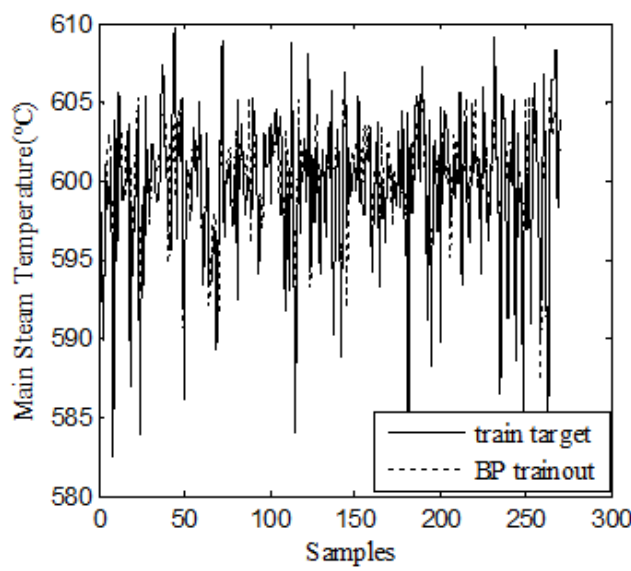

(a) $l=12$, BP train results

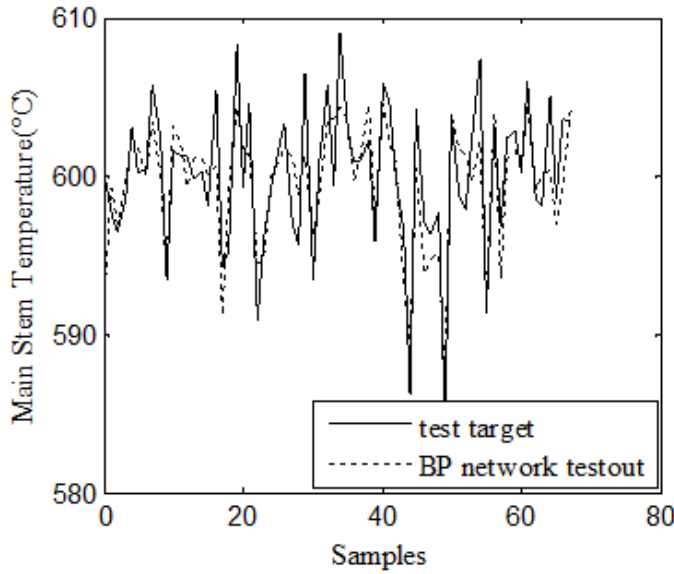

(b) $l=12$, BP test results

Fig.8.BP network train and test performance

g.8 (a) and (b) show the results of modeling based on the chosen activation functions and layer numbers. The selection of numbers and function types has dominant influence on the modeling performance. During training process, BP tracked well and training error changed a little around zero. In predicting, the BP test values experienced ups and downs, but still followed the target. That is to say, either training or predicting output of the built BP model can approximately fit the actual value.

\section{Conclusions}

The BP neural network modeling was established for the main steam temperature of a 1000MW thermal power unit. The number of layers and the number of neurons in the particular layers have been chosen in the experimental way, observing the learning effectiveness for different configurations of the structure of the neural network and different parameters of the network. It was proved that the hidden layer numbers, hidden layer and the activation function of the output layer definitely influence the modeling effect. The model has good generalization and approximation 
performance, and can fit the main temperature variations with the feature parameters under different loads. When the number of hidden nodes and the type of activation function change, the trained network is tested, this can be used as a reference for network type selection. The simulation results show that the proposed method can reveal the main steam temperature with acceptable performances in a large variable load range. Although the BP neural network has a good fitting ability, the results still have some deviation and should be optimized in further research.

\section{Acknowledgements}

This work is supported by National Science Foundation of China (No. U1504617).

\section{References}

[1] Yongping, Yang, Zhiping Yang, Gang $\mathrm{Xu}$ and Ningling Wang. Situation and Prospect of Energy Consumption for China's Thermal Power Generation. Proceedings of CSEE. 2013, 33 (23):5-11.(In Chinese)

[2] Guiquan Fan. Study on Characteristics and Control of the Steam Temperature of Supercritical Boilers.Journal of Power Engineering. 2007, 27(1):34-37. (In Chinese)

[3] Guiquan Fan. Control of Intermediate Point Temperature and Steam Temperature of 1000MW Ultra Supercritical. Journal of Power Engineering. 2007, 27(3):332-335.

[4]Lijia, Zongjun Cai. Neuro-fuzzy Based PID Cascade Control of Main Steam Temperature of Fire Electrical Engineering Set. Control Engineering of China,. 2013, 20(5):877-881. (In Chinese)

[5] Dongfeng Wang, Jinshan Huang. Identification of Main Steam Temperature System Based on Improved Harmony Search Algorithm and Field Data. Journal of Chinese Society of Power Engineering. 2014, 34(5):376-381. (In Chinese)

[6] JingtaoHuang, Huanglong Ma, Jianbo Mao and Jixin Qian. Identification of Main Steam Temperature System Based on Improved Harmony Search Algorithm and Field Data. Proceedings of the CSEE. 2006, 26(7):19-24. (In Chinese)

[7] Dhanuskodi R, Kaliappan R, Suresh S, Anantharaman N, Arunagiri A, Krishnaiah J. Artificial Neural Networks model for predicting wall temperature of supercritical boilers. Applied Thermal Engineering. 2015, 90:749-753.

[8] Ma Y, Yang S, Ca S, Wang Y. The Simulation Research and Neural Network Modeling of Superheated Steam Temperature Characteristics for Ultra-Supercritical Unit. 27th Chinese Control and Decision Conference; Qingdao: Institute of Electrical and Electronics Engineers Inc; 2015, p. 2474-2478.

[9] Wu X, Shen J, Li Y, Lee KY. Data-driven Modelling and Predictive Control for Boiler-turbine Unit Using Fuzzy Clustering and Subspace Methods. ISA Trans. 2014, 53(3):699-708.

[10] WS M, W P. A Logical Calculus of the Ideas Immanent in Nervous Activity.[J]. Bulletin Math Biophys. 1943, (5):33-115. 